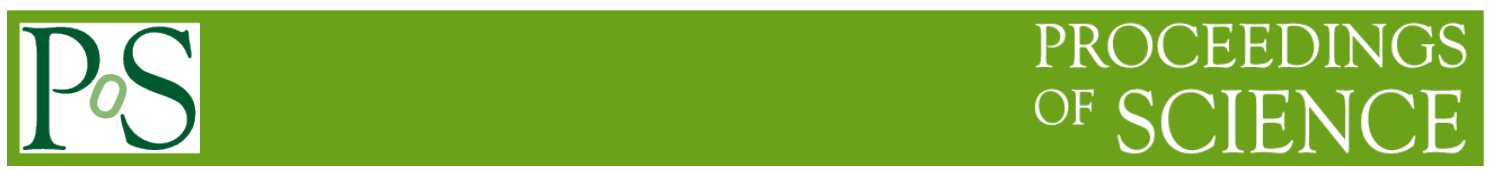

\title{
Nucleon Spin Structure Today and Tomorrow
}

\section{Anatoly Efremov}

Joint Inslitute for Nuclear Research

Dubna, 141980 Russia

E-mail:efremov@theor.jinr.ru

A short review on the theoretical and experimental situation with the Transversal Momentum Dependent parton distriburion functions is given. All the detailes and conclusions can be found in the web site of the Conference http://relnp.jinr.ru/ishepp-xxii/presentations/Efremov.pdf

XXII International Baldin Seminar on High Energy Physics Problems

15-20 September 2014

JINR, Dubna, Russia 